\title{
ASYMPTOTIC BEHAVIOR OF LINEAR INTEGRODIFFERENTIAL SYSTEMS
}

\author{
BY \\ VIOREL BARBU AND STANLEY I. GROSSMAN ${ }^{(1)}$
}

\begin{abstract}
We consider the system (L) $y^{\prime}(t)=A y(t)+\int_{-\infty}^{t} B(t-s) y(s) d s$, $y(t)=f(t), t \leq 0$ where $y(t)$ is an $n$-vector and $A$ and $B(t)$ are $n \times n$ matrices. System (L) generates a semigroup given by $T_{t} f(s)=y(t+s ; f)$ for $f$ bounded, continuous and having a finite limit at $-\infty$. Under hypotheses concerning the roots of $\operatorname{det}(\lambda I-A-\hat{B}(\lambda))$, where $\hat{B}(\lambda)$ is the Laplace transform, various results about the a symptotic behavior of $y(t)$ are derived, generally after invoking the Hille-Yosida theorem. Two typical results are Theorem 1 . If $B(t) \in L 1[0, \infty)$ and $(\lambda I-A-\hat{B}(\lambda))^{-1}$ exists for $\operatorname{Re} \lambda>0$, then for every $\epsilon>0$, there is an $M_{\epsilon}$ such that $\left\|T_{t} f\right\| \leq M_{\epsilon} e^{\epsilon t}\|f\|$. Theorem 2. If $(\lambda I-A-\hat{B}(\lambda))^{-1}$ exists for $\operatorname{Re} \lambda>-a$ $(a>0)$ and if $B(t) e$ at $\in L 1[0, \infty)$, then the solution to $(\mathrm{L})$ is exponentially asymp. totically stable.
\end{abstract}

I. Introduction. In this paper we will consider the linear Volterra integrodifferential system

$$
y^{\prime}(t)=A y(t)+\int_{-\infty}^{t} B(t-s) y(s) d s ; \quad y(t)=f(t), \quad t \leq 0,
$$

where $y$ is an $n$-vector, $A$ is an $n \times n$ matrix, $B$ an $n \times n$ matrix of functions and $f(t)$ is an $n$-vector of functions lying in an appropriate Banach space.

In [1] and [2] Miller and Grossman considered the case of a finite lag (i.e. $-\infty$ is replaced by $-\tau$ ). The principal result found in [2] was that if $B(t) \in L^{1}[0, \infty)$ then asymptotic stability of the solution was equivalent to the condition $\operatorname{det}(\lambda I-A-\hat{B}(\lambda)) \neq 0$ for $\operatorname{Re} \lambda \geq 0$ where $\hat{B}(\lambda)$ denotes the Laplace transform.

In the present work we exploit semigroup properties of the solutions to (L) which yield results about their asymptotic behavior. We give, for example, conditions under which the solution of $(\mathrm{L})$ is exponentially asymptotically stable (Corollary 3.3). Under milder restrictions, we show (Corollary 3.6) that solutions can have at most logarithmic growth and in Theorem 3.8 we give conditions under which the solution of (L) lies in $L^{2}[0, \infty)$.

The authors have benefited from much fruitful correspondence with Professor A. Halanay in Bucharest.

Presented to the Society, January 17, 1972; received by the editors October 29, 1971.

AMS (MOS) subject classifications (1969). Primary 3450, 3475, 4513, 4750.

Key words and phrases. Volterra integrodifferential systems, infinite lag, exponential asymptotic stability, logarithmic growth, solutions in $L 2[0, \infty),\left(C_{0}\right)$ semigroup, resolvent of $\left(C_{0}\right)$ semigroup, Hardy class $H^{2}$.

(1) Research supported in part by the National Research Council of Canada under grant \#A7337. 
II. Preliminaries.

Definition 2.1. Let $X$ be a Banach space. Let $\left\{T_{t}\right\}, 0 \leq t<\infty$, be a family of operators taking $X$ into itself. (1) $\left\{T_{t}\right\}$ is a semigroup if $T_{0} f=f$ and $T_{t+s}(f)$ $=T_{t}\left(T_{s}(f)\right)$. (2) The infinitesimal generator of $T_{t}$ is defined as $C f=$ $\lim _{t \rightarrow 0}\left(T_{t} f-f\right) / t$ whenever that limit exists and the set of those $f \epsilon X$ for which the limit exists is the domain of $C(D(C))$. (3) $\left\{T_{t}\right\}$ is said to be a $\left(C_{0}\right)$ semigroup if, for each $f \in X, \lim _{t \rightarrow 0} T_{t} f-f=0$.

The following theorem is a summary of results which can be found in [3].

Theorem 2.1. If $T_{t}$ is a $C_{0}$ semigroup, then $D(C)$ is dense in $X$; and the range of $C$ is contained in $X$. Furthermore, for $x \in D(C),(d / d t) T_{t} x=C T_{t} x=$ $T_{t} C x$.

Now, let $X$ be the space $B C_{l}(-\infty, 0]$, i.e. $x \in X$ if and only if $x(t)$ is bounded and continuous in $(-\infty, 0]$ and has finite limit at $-\infty$. Suppose $B(t) \epsilon$ $L^{1}[0, \infty)$. Then we can rewrite system $(L)$ as

$$
y^{\prime}(t)=A y(t)+\int_{-\infty}^{0} B(t-s) f(s) d s+\int_{0}^{t} B(t-s) y(s) d s .
$$

The first integral above is clearly bounded for every $t \geq 0$ and $f \in X$. It is then possible to conclude (see Driver [4] for details) that there is a unique solution to (L) which is continuable to $+\infty$ (i.e. has no finite escape time). We now define a set of operators $\left\{T_{t}\right\}$ as $T_{t} f(s) \equiv y(t+s ; f)$, where $y(\cdot ; f)$ is the solution of (L) with initial function $f$. Clearly $T_{t} f \in B C_{l}(-\infty, 0]$ if $f$ does.

Lemma 2.2. $\left\{T_{t}\right\}$ is a $C_{0}$ semigroup of operators mapping $X \rightarrow X$. The infinitesimal generator $C f$ is given by

$$
\begin{gathered}
C f(s)=d f / d s, \quad s \leq 0, \\
D(C)=\left\{f: f^{\prime \prime} \in B C_{l}(-\infty, 0] \text { and } f^{\prime}(0)=A f(0)+\int_{-\infty}^{0} B(-s) f(s) d s\right\} .
\end{gathered}
$$

In order to prove this lemma, we need to define a solution to (L).

Definition 2.2. A function $y(t ; f)$ is a solution to (L) if and only if $y(t ; f)$ is continuous for $-\infty<t<\infty$, differentiable for $t>0$ and $y(t ; f)=f(t)$ for $t \leq 0$.

Proof of Lemma 2.2. We show that $T_{v+u} f=T_{v}\left(T_{u} f\right), u, v \geq 0$. (By definition it is clear that $T_{0} f=f$.) From the definition of a solution,

$$
\begin{gathered}
y^{\prime}(v+u ; f)=A y(v+u ; f)+\int_{0}^{v+u} B(v+u-s) y(s ; f) d s+\int_{-\infty}^{0} B(v+u-s) f(s) d s \\
=A y(v+u ; f)+\int_{-u}^{v} B(v-\sigma) y(\sigma+u ; f) d \sigma+\int_{-\infty}^{-u} B(v-\sigma) f(\sigma+u) d \sigma \\
=A y(v+u ; f)+\int_{0}^{v} B(v-\sigma) y(\sigma+u ; f) d \sigma+\int_{-\infty}^{0} B(v-\sigma) y(\sigma+u ; f) d \sigma .
\end{gathered}
$$


Let $z(v) \stackrel{\text { def }}{=} y(v+u ; f)$. Then $z(v)=y(v ; y(u+\cdot ; f))$ which implies that $y(v+u ; f)$ $=y\left(v ; y(u+\cdot ; f)\right.$ or, equivalently, $T_{v+u} f=T_{v}\left(T_{u} f\right)$. The fact that $\left\{T_{t}\right\}$ is a $C_{0}$ semigroup follows easily from the uniform continuity of functions in $B C_{l}(-\infty, 0]$. Finally, the infinitesimal generator $C f(s)=\lim _{t \rightarrow 0^{+}}\left(T_{t} f-T_{0} f\right) / t=$ $\lim _{t \rightarrow 0^{+}}(f(t+s)-f(s)) / t=f^{\prime}(s)$, for $s<0$. For $s=0$, we have $\lim _{t \rightarrow 0}(y(t ; f)-y(0 ; f)) / t$ $=y^{\prime}(0 ; f)=A f(0)+\int_{-\infty}^{0} B(-s) f(s) d s$ and the proof is complete.

We now calculate the resolvent $R(\lambda, C)$ of the infinitesimal generator. We have $R(\lambda, C) g=(\lambda I-C)^{-1} g=f$ or $(\lambda I-C) f=g$. Hence, by (2.1) and (2.2), we have

$$
\lambda f(s)-f^{\prime}(s)=g(s), \quad s<0,
$$

and

$$
\lambda f(0)-A f(0)-\int_{-\infty}^{0} B(-\sigma) f(\sigma) d \sigma=g(0) .
$$

Solving the linear, inhomogeneous equation (2.3), we have

$$
f(\sigma)=e^{\lambda \sigma} f(0)-\int_{0}^{\sigma} e^{\lambda(\sigma-\tau)} g(\tau) d \tau, \quad \sigma \leq 0 .
$$

But, from (2.4),

$$
\lambda f(0)-A f(0)-\int_{-\infty}^{0} B(-\sigma)\left[e^{\lambda \sigma_{f}(0)}-\int_{0}^{\sigma} e^{\lambda(\sigma-\tau)} g(\tau) d \tau\right] d \sigma=g(0)
$$

Then

$$
\begin{aligned}
{\left[\lambda I-A-\int_{-\infty}^{0} B(-\sigma) e^{\lambda \sigma} d \sigma\right] f(0) } \\
\quad=g(0)-\int_{-\infty}^{0} B(-\sigma)\left[\int_{0}^{\sigma} e^{\lambda(\sigma-\tau)} g(\tau) d \tau\right] d \sigma
\end{aligned}
$$

which implies that

$$
f(0)=(\lambda I-A-\hat{B}(\lambda))^{-1}\left\{g(0)-\int_{-\infty}^{0} B(-\sigma)\left[\int_{0}^{\sigma} e^{\lambda(\sigma-\tau)} g(\tau) d \tau\right] d \sigma\right\} .
$$

This holds whenever $(\lambda I-A-\hat{B}(\lambda))^{-1}$ exists, i.e. for $\operatorname{Re} \lambda$ large enough. Here $\hat{B}(\lambda)$ denotes the Laplace transform.

Finally, we have

$$
\begin{aligned}
(R(\lambda, C) g)(s)= & e^{\lambda s}(\lambda I-A-\hat{B}(\lambda))^{-1} g(0) \\
& +e^{\lambda s}(\lambda I-A-\hat{B}(\lambda))^{-1} \int_{0}^{\infty} e^{-\lambda t} \int_{-\infty}^{0} B(t-s) g(s) d s d t \\
& +e^{\lambda s} \int_{s}^{0} e^{-\lambda t} g(t) d t .
\end{aligned}
$$


We note that all the above computations are justified for $\operatorname{Re} \lambda>\omega$ for $\omega$ large enough and using the fact that $B(t) \in L^{1}[0, \infty)$. Also, the resolvent could have calculated from the well-known formula (see [3])

$$
(R(\lambda, C) g)(s)=\int_{0}^{\infty} e^{-\lambda t}\left(T_{t} g\right)(s) d t \quad \operatorname{Re} \lambda>\omega .
$$

Finally, we can point out that all $\lambda$ with $\operatorname{Re} \lambda<0$ lie in the spectrum of $C$. This is evident from the fact that even if $(\lambda I-A-\hat{B}(\lambda))^{-1}$ exists (so that equation (2.5) makes sense), the function $e^{\lambda s}$ is unbounded on $(-\infty, 0]$ and is, therefore, not a member of $B C_{l}(-\infty, 0]$. This limits the results obtainable from pure semigroup theory. For example, in order to derive exponential stability results directly, we would need to have the semigroup $\left\{T_{t}\right\}$ of type $-a$, where $a>0$. For this condition, a minimal requirement would be that the spectrum of $R$ lies to the left of the line $\operatorname{Re} \lambda=-\alpha$, a fact contradicted above.

To conclude this section, we mention some results about the type of a semigroup.

Definition 2.3. We say that $T_{t}$ is of type $\omega_{0}$ if and only if

$$
\omega_{0}=\inf _{t>0} \frac{1}{t} \log \left\|T_{t}\right\|=\lim _{t \rightarrow \infty} \frac{1}{t} \log \left\|T_{t}\right\| \text {. }
$$

(See [3, p. 306].) It is clear that if $\left\{T_{t}\right\}$ is of type $\omega_{0}$, then there is a constant $M_{\omega_{0}}$ such that $\left\|T_{t} x\right\| \leq M_{\omega_{0}} e^{\omega_{0} t}\|x\|$ for every $x \in X$.

Theorem (Hille-Yosida). A necessary and sufficient condition that a closed linear operator $C$ generates a semigroup $T_{t}$ of class $\left(C_{0}\right)$ and of type $\omega_{0}$ is that $D(C)$ be dense in $X$ and $\| R(\lambda, C)]^{n} \| \leq M_{\omega_{0}} /\left(\lambda-\omega_{0}\right)^{n}$ for $\lambda>\omega_{0}$.

Note. From the resolvent equations, this is equivalent to

$$
\left\|d^{n} R(\lambda, C) / d \lambda^{n}\right\| \leq M_{\omega_{0}} n ! /\left(\lambda-\omega_{0}\right)^{n+1}
$$

since $R(\lambda, C)=R(\mu, C)+(\mu-\lambda) R(\lambda, C) R(\mu, C)$, which implies that $\left(d^{n} / d \lambda^{n}\right) R(\lambda, C)$ $=(-1)^{n} n ! R(\lambda, C)^{n+1}$.

It is not necessary to only consider this semigroup in the space $B C_{l}(-\infty, 0]$. An easy alternative is to consider the space $X=B C_{l}(-\infty, 0] \cap L^{p}(-\infty, 0]$ with $\|x\|_{X}=\|x\|_{\infty}+\|x\|_{L} p \cdot X$ is a Banach space with this norm and it is evident that $\left\{T_{t}\right\}$ defined above is again a strongly continuous semigroup of operators. This follows easily from the fact that the convolution of an $L^{1}$ function with an $L^{p}$ function is again in $L^{p}$. The infinitesimal generator of $\left\{T_{t}\right\}$ takes the same form, the only change being that now

$$
D(C)=\left\{f: f^{\prime} \in B C_{l}(-\infty, 0] \cap L^{p}(-\infty, 0] \text { and } f^{\prime}(0)=A f(0)+\int_{-\infty}^{0} B(-s) f(s) d s\right\} .
$$


We should point out again that the hypotheses of continuity and boundedness of $f$ together with $B(t) \in L^{1}[0, \infty)$ ensure that there is always a unique continuous solution to (L) existing for $-\infty<t<\infty$. Moreover, from Lemma 2.2, we can conclude that there is an $\omega>0$ such that

$$
\left\|T_{t} f\right\| \leq M e^{\omega t}\|f\|, \text { for every } t>0 .
$$

This means that $|y(t ; f)| \leq M e^{\omega t}\|f\|$ where $y(t ; f)$ is the unique solution to (L).

III. The main results. Our first results concern the space $X=B C_{l}(-\infty, 0] \cap$ $L^{1}(-\infty, 0]$. Generalization to the $L^{p}$ case is immediate.

Theorem 3.1. Let $B(t) \in L^{1}[0, \infty)$ and suppose that

(i) $(\lambda I-A-\hat{B}(\lambda))^{-1}$ exists for $\operatorname{Re} \lambda>0$, and

(ii) $\left\|\left(d^{n} / d \lambda^{n}\right)(\lambda I-A-\hat{B}(\lambda))^{-1}\right\|<M n ! / \lambda^{n+1}$ for $\lambda$ large enough.

Then, for every $\epsilon>0$, there exists $M_{\epsilon}>0$ such that

$$
\left\|T_{t} f\right\|_{X} \leq M_{\epsilon} e^{\epsilon t}\|f\|_{X} \text { for } t \geq 0 .
$$

Proof. From the Hille-Yosida theorem, it suffices to prove that, for every $\epsilon>0$, there is an $M_{\epsilon}>0$ such that $\left\|d^{n} R(\lambda, C) / d \lambda^{n}\right\|_{X} \leq M_{\epsilon} n ! /(\lambda-\epsilon)^{n+1}$. To show this we first note that

$$
\begin{aligned}
& \left\|\frac{d^{n}}{d \lambda^{n}} e^{\lambda s}(\lambda I-A-\hat{B}(\lambda))^{-1} g(0)\right\|_{\infty} \\
& \quad \leq\|g\|_{\infty}\left\|\sum_{k=0}^{n}\left(\begin{array}{l}
n \\
k
\end{array}\right) s^{k} e^{\lambda s} \frac{M(n-k) !}{\lambda^{n-k}+1}\right\| \leq\|g\|_{\infty} \frac{M n !}{\lambda^{n+1}}\left\|\sum_{k=0}^{n} \frac{s^{k} \lambda^{k}}{k !}\right\| \cdot\left\|e^{\lambda s}\right\|_{\infty} \\
& \quad \leq \frac{M n !}{\lambda^{n+1}}\|g\|_{\infty} \leq \frac{M n !}{\lambda^{n+1}}\left(\|g\|_{\infty}+\|g\|_{1}\right)=\frac{M n !}{\lambda^{n+1}}\|g\|_{X} .
\end{aligned}
$$

Similarly, consider

$$
\begin{gathered}
\left\|\frac{d^{n}}{d \lambda^{n}} e^{\lambda s}(\lambda I-A-\hat{B}(\lambda))^{-1} \int_{0}^{\infty} e^{-\lambda t} \int_{-\infty}^{0} B(t-s) g(s) d s d t\right\|_{\infty} \\
\leq M\|B\|_{1}\|g\|_{\infty}\left\|\sum_{k=0}^{n}\left(\begin{array}{l}
n \\
k
\end{array}\right) \frac{k !}{\lambda^{k+1}} \int_{0}^{\infty} t^{n-k} e^{-\lambda t} d t\right\| .
\end{gathered}
$$

For $\epsilon>0,1 / \lambda^{k+1}<1 /(\lambda-\epsilon)^{k+1}$. After multiplying and dividing by $(\lambda-\epsilon)^{n-k}$, (3.2) becomes 


$$
\begin{aligned}
& \leq \frac{M\|B\|_{1}\left\|_{g}\right\|_{\infty} n !}{(\lambda-\epsilon)^{n+1}}\left\|\sum_{k=0}^{n} \int_{0}^{\infty} e^{-(\lambda-\epsilon) t} \frac{(t(\lambda-\epsilon))^{n-k}}{(n-k) !} e^{-\epsilon t} d t\right\| \\
& =\frac{M_{1} n !\|g\|_{\infty}}{(\lambda-\epsilon)^{n+1}}\left\|\int_{0}^{\infty} e^{-(\lambda-\epsilon) t}\left(\sum_{k=0}^{n} \frac{(t(\lambda-\epsilon))^{n-k}}{(n-k) !}\right) e^{-\epsilon t} d t\right\| \\
& \left.\leq \frac{M_{1} n !\|g\|_{\infty}}{(\lambda-\epsilon)^{n+1}} \int_{0}^{\infty} e^{-\epsilon t} d t \leq \frac{M_{1} n ! / \epsilon}{(\lambda-\epsilon)^{n+1}}\|g\|_{X} \quad \text { (where } M_{1}=M\|B\|_{1}\right) .
\end{aligned}
$$

Finally

$$
\begin{gathered}
\left\|\frac{d^{n}}{d \lambda^{n}} \int_{s}^{0} e^{\lambda(s-t)} g(t) d t\right\| \leq\|g\|_{\infty}\left\|\int_{s}^{0}(s-t)^{n} e^{\lambda(s-t)} d t\right\| \\
\leq\|g\|_{\infty}\left\|\int_{-\infty}^{0} u^{n} e^{\lambda u} d u\right\| \leq \frac{n !}{\lambda^{n+1}}\|g\|_{X} .
\end{gathered}
$$

Hence we have

$$
\left\|\left(d^{n} / d \lambda^{n}\right) R(\lambda, C) g\right\|_{\infty} \leq\left(M_{\epsilon} n ! /(\lambda-\epsilon)^{n+1}\right)\|g\|_{X} .
$$

We now prove the same thing for $\left\|\left(d^{n} / d \lambda^{n}\right) R(\lambda, C) g\right\|_{1}$. Now,

$$
\begin{aligned}
& \| \int_{-\infty}^{0} \frac{d^{n}}{d \lambda^{n}} e^{\lambda s}(\lambda I-A-\hat{B}(\lambda))^{-1} g(0) d s \mid \\
& \quad \leq M\|g\|_{\infty}\left|\int_{-\infty}^{0} \sum_{k=0}^{n}\left(\begin{array}{l}
n \\
k
\end{array}\right) e^{\lambda s} s^{n-k} \frac{k !}{\lambda^{k+1}}\right|=M n !\|g\|_{\infty}\left|\sum_{k=0}^{n} \int_{-\infty}^{0} e^{\lambda s} \frac{s^{n-k} d s}{(n-k) ! \lambda^{k+1}}\right| \\
& \quad \leq \frac{M n !\|g\|_{\infty}}{(\lambda-\epsilon)^{n+1}}\left|\int_{-\infty}^{0} e^{(\lambda-\epsilon) s}\left(\sum_{k=0}^{n} \frac{(s(\lambda-\epsilon))^{n-k}}{(n-k) !}\right) e^{\epsilon s} d s\right| \\
& \quad \leq\left(M n ! / \epsilon(\lambda-\epsilon)^{n+1}\right)\left\|_{g}\right\|_{X} \text { as before. }
\end{aligned}
$$

Similarly,

$$
\begin{aligned}
& \left|\int_{-\infty}^{0} \frac{d^{n}}{d \lambda^{n}} e^{\lambda s}(\lambda I-A-\hat{B}(\lambda))^{-1} \int_{0}^{\infty} e^{-\lambda t} \int_{-\infty}^{0} B(t-u) g(u) d u d t d s\right| \\
& \quad \leq M\|B\|_{1}\|g\|_{X}\left|\int_{-\infty}^{0} \sum_{k=0}^{n}\left(\begin{array}{l}
n \\
k
\end{array}\right) \sum_{r=0}^{k}\left(\begin{array}{l}
k \\
r
\end{array}\right) \frac{r !}{\lambda^{r+1}} e^{\lambda s} s^{k-r} \int_{0}^{\infty} t^{n-k} e^{-\lambda t} d t d s\right|
\end{aligned}
$$

which, using the same method as before, is

$$
\leq\left(M_{1} n ! / \epsilon^{2}(\lambda-\epsilon)^{n+1}\right)\|g\|_{X} \text {. }
$$


Finally, consider

$$
\left|\int_{-\infty}^{0} \frac{d^{n}}{d \lambda^{n}} \int_{s}^{0} e^{\lambda(s-t)} g(t) d t d s\right|=\left|\int_{-\infty}^{0}\left[\int_{s}^{0}(s-t)^{n} e^{\lambda(s-t)} g(t) d t\right] d s\right| .
$$

Let $f(t)=t^{n} e^{\lambda t}$. Then the above integral is equal to the $L^{1}(-\infty, 0]$ norm of the convolution of $f$ and $g$. But

$$
\|f * g\|_{1} \leq\|f\|_{1}\|g\|_{1} \leq\left(n ! / \lambda^{n+1}\right)\|g\|_{X} \text {. Q.E.D. }
$$

Corollary 3.2. Suppose that $(\lambda I-A-\hat{B}(\lambda))^{-1}$ exists for $\operatorname{Re} \lambda>0$ and that $(\lambda I-A)^{-1}$ exists for $\operatorname{Re} \lambda \geq 0$. Suppose further that $\left\|e^{A t}\right\|_{L_{1}[0, \infty)} \cdot\|B\|_{L_{1}[0, \infty)}<1$. Then the conclusions of Theorem 3.1 hold.

Proof. We must verify hypothesis (ii) of that theorem. We have

$$
\begin{aligned}
(\lambda I-A-\hat{B}(\lambda))^{-1} & =(\lambda I-A)^{-1}\left(I-(\lambda I-A)^{-1} \hat{B}(\lambda)\right)^{-1} \\
& =(\lambda I-A)^{-1} \sum_{n=0}^{\infty}\left[(\lambda I-A)^{-1} \hat{B}(\lambda)\right]^{n}
\end{aligned}
$$

which converges by assumption. Also, $(\lambda I-A)^{-1}=\int_{0}^{\infty} e^{-\lambda s} e^{A s} d s$ and $\hat{B}(\lambda)=$ $\int_{0}^{\infty} e^{-\lambda t} B(t) d t$. And so $(\lambda I-A)^{-1} \hat{B}(\lambda)=\int_{0}^{\infty} \int_{0}^{\infty} e^{-\lambda(s+t)} e^{A s} B(t) d s d t$. After a change of variables and a change in the order of integration, we obtain

$$
(\lambda I-A)^{-1} \hat{B}(\lambda)=\int_{0}^{\infty} \int_{0}^{s} e^{-\lambda u} e^{A(u-t)} B(t) d t d u=\widehat{e^{A t} * B(\lambda)}
$$

where " $*$ " denotes convolution. Consequently, $\left[(\lambda I-A)^{-1} \hat{B}(\lambda)\right]^{n}=$ the Laplace transform of $\left(e^{A t} * B\right) *\left(e^{A t} * B\right) * \ldots *\left(e^{A t} * B\right)$ (n times). Denote this function by $G_{n}(t)$. Then

$$
\frac{d^{k}}{d \lambda^{k}}\left[(\lambda I-A)^{-1} \hat{B}(\lambda)\right]^{n}=\frac{d^{k}}{d \lambda^{k}} \int_{0}^{\infty} e^{-\lambda t} G_{n}(t) d t=\int_{0}^{\infty} e^{-\lambda t} t^{k} G_{n}(t) d t .
$$

But, by integration by parts, it is easy to show that this last expression is bounded by $\left(k ! / \lambda^{k+1}\right) \sup _{0 \leq t<\infty}\left|G_{n}(t)\right|$. From the equality $G_{n}(t)=\int_{0}^{\infty} G_{n-1}(t-s)\left[\left(e^{A(t)} * B\right)(s)\right] d s$, we obtain $\left\|G_{n}\right\|_{\infty} \leq\left\|G_{n-1}\right\|_{\infty}\left\|e^{A t} * B\right\|_{1} \leq\left\|e^{A t}\right\|_{1}\|B\|_{1}\left\|G_{n-1}\right\|_{\infty}$. Hence $\left\|G_{n}\right\|_{\infty} \leq$ $\left(\left\|e^{A t}\right\|_{1}\|B\|_{1}\right)^{n}$. Thus $\left(d^{k} / d \lambda^{k}\right) \sum_{n=0}^{\infty}\left[(\lambda I-A)^{-1} \hat{B}(\lambda)\right]^{n} \leq M_{1} k ! / \lambda^{k+1}$ where $M_{1}=$ $1 /\left(1-\left\|e^{A t}\right\|_{1}\|B\|_{1}\right)$. Since $\left|\left(d^{k} / d \lambda^{k}\right)(\lambda I-A)^{-1}\right| \leq M_{2} k ! / \lambda^{k+1}$, the result follows.

The next corollary gives conditions which ensure exponential asymptotic stability of the solution.

Corollary 3.3. Suppose that $e^{a t} B(t) \in L^{1}[0, \infty)$ for some $a>0$, condition (ii) of Theorem 3.1 is satisfied, and condition (i) bolds for $\operatorname{Re} \lambda>-\alpha$. Then for any $\beta, 0<\beta<a$, there exists $M_{\beta}>0$ such that for any $f \in B C_{l}(-\infty, 0],|y(t ; f)| \leq$ $M_{\beta} e^{-(\alpha-\beta) t}\|f\|_{X}$. In particular, $y(t ; f) \in L^{1}(-\infty, \infty)$. 
Proof. Let $z(t ; f)=e^{a_{t}} y(t ; f)$; then $y(t)=e^{-a_{t}} z(t)$ and

$$
\begin{aligned}
y^{\prime}(t) & =-\alpha e^{-\alpha t} z(t)+e^{-\alpha t} z^{\prime}(t) \\
& =A y(t)+\int_{0}^{t} B(t-s) y(s) d s+\int_{-\infty}^{0} B(t-s) f(s) d s .
\end{aligned}
$$

Hence $z^{\prime}(t)=(\alpha I+A) z(t)+\int_{0}^{t} B_{\alpha}(t-s) z(s) d s+\int_{-\infty}^{0} B_{\alpha}(t-s) f_{\alpha}(s) d s$ where $B_{\alpha}(t)$ $=e^{\alpha t} B(t)$ and $f_{a}(t)=e^{\alpha_{t}} f(t) \in B C_{l}(-\infty, 0]$ since $f$ does. Now, $\hat{B}_{\alpha}(\lambda)=\hat{B}(\lambda-\alpha)$ and $\operatorname{det}\left[\lambda I-(A+\alpha I)-\hat{B}_{\alpha}(\lambda)\right]=\operatorname{det}[(\lambda-\alpha) I-A-\hat{B}(\lambda-\alpha)]$ which is nonzero for $\operatorname{Re} \lambda>0$. Thus, for $0<\beta<\alpha$, there exists $M_{\beta}$ such that $\left|z\left(t ; f_{\alpha}\right)\right| \leq M_{\beta} e^{\beta t}\left\|f_{\alpha}\right\|_{X}$ $\leq M_{\beta} e^{\beta t}\|f\|_{X}$. Q.E.D.

It should be noted here that exponential asymptotic stability is, even for the linear case, not implied by asymptotic stability. In [5], for example, Levin and Nohel give an asymptotically stable, linear system where solutions go to zero as $t^{-3 / 2}$. Thus, exponential stability results are somewhat of a rarity in this theory.

The following converse theorem is true. The proof is essentially the one given by Miller in [1].

Theorem 3.4. Suppose the conclusion of Theorem 3.1 bolds. Then $\operatorname{det}(\lambda I-A-\hat{B}(\lambda)) \neq 0$ for $\operatorname{Re} \lambda>0$.

Proof. Suppose not. Then there exists a complex number $s_{0}$ and a unit vector $k_{0}$ such that $\operatorname{Re} s_{0}>0$ and $\left(s_{0} I-A-\hat{B}\left(s_{0}\right)\right) x_{0}=0$. Define $x(t)=e^{s_{0} t} x_{0}$. Clearly $x(t)$ is bounded on $-\infty<t \leq 0$ and $x^{\prime}(t)-A x(t)-\int_{-\infty}^{t} B(t-s) x(s) d s=$ $\left(s_{0} I-A-\hat{B}\left(s_{0}\right)\right) x_{0} e^{s_{0} t}=0$. But for $\epsilon<\operatorname{Re} s_{0}$, the conclusions of Theorem 3.1 are not valid. Q.E.D.

For the case $X=B C_{l}(-\infty, 0]$, it is possible to remove hypothesis (ii) from Theorem 3.1.

Theorem 3.5. Suppose that $\operatorname{det}(\lambda I-A-\hat{B}(\lambda)) \neq 0$ for $\operatorname{Re} \lambda>0$, and $B(t) \epsilon$ $L^{1}[0, \infty)$. Then for every $\epsilon>0$, there is an $M_{\epsilon}>0$ such that $\left\|T_{t}\right\|_{\infty} \leq M_{\epsilon} e^{\epsilon t}\|f\|_{\infty}$.

Proof. Since $R(\lambda, C)$ is a "Laplace transform" (see 2.6), we can use the well-known inversions formula

$$
T_{t} f=\frac{1}{2 \pi i} \int_{\omega-i \infty}^{\omega+i \infty} e^{\lambda t} R(\lambda, C) f d \lambda, \quad t>0, \quad \omega \text { sufficiently large. }
$$

This is valid for every $f \in D(C)$. By the definition of $T_{t}$ we have, in particular, that

$$
y(t)=\left(T_{t} f\right)(0)=\frac{1}{2 \pi i} \int_{\omega-i \infty}^{\omega+i \infty} e^{\lambda t}(R(\lambda, C) f)(0) d \lambda, \quad t>0 .
$$

From our assumptions and (2.5) we see that $R(\lambda, C) f$ is analytic in the whole halfplane $\operatorname{Re} \lambda>0$. Moreover, 


$$
(R(\lambda, C) f)(0)=(\lambda I-A-\hat{B}(\lambda))^{-1}(f(0)+\widehat{B * f}(\lambda))
$$

We can write

$$
\begin{aligned}
\lambda(R(\lambda, C) f)(0)= & f(0)+\widehat{B * f}(\lambda) \\
& +(A+\hat{B}(\lambda))(\lambda I-A-\hat{B}(\lambda))^{-1}(f(0)+\widehat{f * B}(\lambda))
\end{aligned}
$$

which holds for $\operatorname{Re} \lambda>0$. Using (3.4) in (3.3), we obtain

$$
\begin{aligned}
y(t)= & \frac{1}{2 \pi i} \int_{\omega-i \infty}^{\omega+i \infty} e^{\lambda t} \frac{f(0)}{\lambda} d \lambda+\frac{1}{2 \pi i} \int_{\omega-i \infty}^{\omega+i \infty} e^{\lambda t} \frac{\widehat{B * f}(\lambda)}{\lambda} d \lambda \\
& +\frac{1}{2 \pi i} \int_{\omega-i \infty}^{\omega+i \infty} e^{\lambda t} \frac{(A+\hat{B}(\lambda))(\lambda I-A-\hat{B}(\lambda))^{-1}(f(0)+\widehat{f * B}(\lambda)) d \lambda}{\lambda} .
\end{aligned}
$$

Hence, (see [3, Theorem 6.3.1])

$$
\begin{aligned}
y(t)= & f(0)+\int_{0}^{t}(\widehat{B * f})(s) d s \\
& +\frac{1}{2 \pi i} \int_{\omega-i \infty}^{\omega+i \infty} e^{\lambda t} \frac{(A+\hat{B}(\lambda))(\lambda I-A-\hat{B}(\lambda))^{-1}(f(0)+\widehat{f * B}(\lambda))}{\lambda}
\end{aligned}
$$

for every $t \geq 0$ and $f \in D(C)$. Let $g(\lambda)=(A+\hat{B}(\lambda))(\lambda I-A-\hat{B}(\lambda))^{-1}(f(0)+\widehat{f * B}(\lambda))$. Since $B \in L^{1}[0, \infty)$ we have $|g(\lambda)| \rightarrow 0$ as $|\operatorname{Im} \lambda| \rightarrow \infty$ uniformly with respect to $\operatorname{Re} \lambda$ in every interval $[\epsilon, \omega], 0<\epsilon<\omega$. Consequently, we can change the contour of integration to obtain

$$
\frac{1}{2 \pi i} \int_{\omega-i \infty}^{\omega+i \infty} e^{\lambda t} \frac{g(\lambda)}{\lambda} d \lambda=\frac{1}{2 \pi} \int_{-\infty}^{\infty} e^{(\epsilon+i \eta) t} \frac{g(\epsilon+i \eta)}{\epsilon+i \eta} d \eta
$$

where $\epsilon>0$ is arbitrarily small. By hypothesis we have (3.6) $\left|(\lambda I-A-\hat{B}(\lambda))^{-1}\right| \leq M /|\lambda|$ for $\operatorname{Re} \lambda>0$ and $|\lambda| \geq r$ for some $r>0$.

We set

$$
\mu(r, \epsilon)=\sup \left\{\left|(\lambda I-A-\hat{B}(\lambda))^{-1}[f(0)+\widehat{B * f}(\lambda)]\right| ; \operatorname{Re} \lambda=\epsilon,|\operatorname{Im} \lambda| \leq r\right\} .
$$

Noting that $|B * f(\lambda)| \leq(1 / \epsilon)\|f\|\|B\|$ for $\operatorname{Re} \lambda=\epsilon$, we have

$$
|g(\epsilon+i \eta)| \leq M_{1}(1+1 / \epsilon)\|f\| \quad \text { if }|\eta| \geq r
$$

and

$$
|g(\epsilon+i \eta)| \leq \mu(r, e) \quad \text { if }|\eta| \leq r
$$

where $M_{1}>0$ is independent of $\epsilon$ and $f$. By using (3.8) and (3.9) we obtain 


$$
\begin{aligned}
& \frac{1}{2 \pi i} \int_{\omega-i \infty}^{\omega+i \infty} e^{\lambda t} \frac{g(\lambda)}{\lambda} d \lambda \\
& \quad \leq \frac{1}{2 \pi} \mu(r, \epsilon) e^{\epsilon t} \int_{|\eta| \leq r} \frac{d \eta}{\sqrt{\eta^{2}+\epsilon^{2}}}+\frac{M_{1}}{2 \pi} e^{\epsilon t^{\prime}}\left(1+\frac{1}{\epsilon}\right)\|f\| \int_{|\eta| \geq r} \frac{d \eta}{\epsilon^{2}+\eta^{2}} .
\end{aligned}
$$

Finally, we have

$$
\begin{aligned}
|y(t)| \leq & |f(0)|+t\|B\|{ }_{1}\|f\|+M e^{\epsilon t(1+1 / \epsilon)\|/\|} \\
& +M \ln \left(\left(r+\sqrt{r^{2}+\epsilon^{2}}\right) / \epsilon\right) \mu(r, \epsilon) e^{\epsilon t} .
\end{aligned}
$$

But

$$
\mu(r, \epsilon) \leq \sup \left\{\left|(\lambda I-A-\hat{B}(\lambda))^{-1}\right|:|\operatorname{Im} \lambda| \leq r, \operatorname{Re} \lambda=\epsilon\right\}\|f\|(1+(1 / \epsilon)\|B\|)
$$

and

$$
\left\|T_{t} f\right\|=\max \left\{\sup _{-\infty<s \leq 0}|f(s)|, \sup _{0 \leq s \leq t}|y(s)|\right\}
$$

Hence

$$
\left\|T_{t} f\right\| \leq M_{\epsilon} e^{\epsilon t}\|f\|, \quad \forall f \in D(C), t>0 .
$$

Since $D(C)$ is dense in $B C_{l}(-\infty, 0]$, the inequality (3.11) can be extended to all of $B C_{l}(-\infty, 0]$ and the proof is complete.

Corollary 3.6. Under the assumptions of Theorem 3.5 for $\operatorname{Re} \lambda \geq 0$, the solution $y(t ; f)$ has, at most, linear growth. If, in addition, $f \in L^{1}(-\infty, 0]$, the growth is logarithmic.

Proof. The first conclusion follows from (3.10) since $|\mu(r, \epsilon)|<M\|f\|(1+(1 / \epsilon)\|B\|)$ and letting $t=1 / \epsilon$. If $f \in L^{1}(-\infty, 0]$, then $\left\|\int_{0}^{t} \widehat{B * f}(s) d s\right\|_{\infty} \leq\left\|B_{1}\right\| f \|_{1}$ and $\widehat{B * f}(\lambda) \leq\|B\|_{1}\|f\|_{1}$. Thus the linear terms become constants and we are left with logarithmic bounds. Q.E.D.

Corollary 3.7. If $e^{a t} B(t) \in L^{1}[0, \infty)$ and $\operatorname{det}(\lambda I-A-\hat{B}(\lambda))^{-1} \neq 0$ for $\operatorname{Re} \lambda>$ $-a$, then the solution of $(\mathrm{L})$ in $B C_{l}(-\infty, 0]$ is exponentially asymptotically stable.

Proof. Same as Corollary 3.3.

We now seek conditions under which the solution of $(L)$ lies in $L^{2}[0, \infty)$. For this we need to introduce the Hardy class $H^{2}$.

Definition 3.1. The function $f(\epsilon+i \eta)$ belongs to $H^{2}$ if and only if $f$ is analytic and $\sup _{\epsilon>0} \int_{-\infty}^{\infty}|f(\epsilon+i \eta)|^{2} d \eta<\infty$. This is a Banach space with the obvious norm. 
Theorem 3.8. Suppose that

(i) $\operatorname{det}(\lambda I-A-\hat{B}(\lambda)) \neq 0$ for $\operatorname{Re} \lambda \geq 0$,

(ii) $B \in L^{1}[0, \infty)$.

Then for every $f \in L^{1}(-\infty, 0] \cap B C_{l}(-\infty, 0]$, the solution $y(t ; f)$ of $(\mathrm{L})$ belongs to $L^{2}[0, \infty)$ and $\|y\|_{2} \leq M\left(\|f\|_{\infty}+\|f\|_{1}\right)$.

Proof. Assumption (i) implies that there is $R>0$ such that

$$
\left|(\lambda I-A-\hat{B}(\lambda))^{-1}\right| \leq M /|\lambda| \text { for }|\lambda| \geq R, \operatorname{Re} \lambda \geq 0
$$

and

$$
\left|(\lambda I-A-\hat{B}(\lambda))^{-1}\right| \leq M \quad \text { for }|\lambda| \leq R, \operatorname{Re} \lambda \geq 0 .
$$

Since $(\lambda I-A-\hat{B}(\lambda))^{-1}$ is analytic in the right halfplane, this implies that $(\lambda I-A-\hat{B}(\lambda))^{-1}$ belongs to $H^{2}$. Consequently,

$$
(\lambda I-A-\hat{B}(\lambda))^{-1}(f(0)+B * f(\lambda)) \epsilon H^{2}
$$

also. This implies (see $[6$, p. 163 , Theorem 2$]$ ) that

$$
\operatorname{l.i.m.~}_{n \rightarrow \infty} \int_{-n}^{n} e^{i \eta t}(\eta i I-A-\hat{B}(i \eta))^{-1}(f(0)+\widehat{B * f}(i \eta)) d \eta=g(t)
$$

exists and lies in $L^{2}[0, \infty)$. But by (3.3)

$$
y(t)=\frac{1}{2 \pi} \lim _{n \rightarrow \infty} \int_{-n}^{n} e^{(\epsilon+i \eta) t} R(\epsilon+i \eta, C)(f)(0) d \eta, \quad t>0 .
$$

Thus, by the Cauchy integral theorem, we have

$$
\begin{aligned}
& \int_{-n}^{n} e^{(\epsilon+i \eta) t} R(\epsilon+i \eta, C)(f)(0) d \eta \\
& (3.16)=\int_{-n}^{n} e^{i \eta t} R(i \eta, C)(f)(0) d \eta \\
& \quad+\int_{0}^{\epsilon} e^{(\sigma+i \eta) t} R(\sigma+i \eta, C)(f)(0) d \sigma-\int_{0}^{\epsilon} e^{(\sigma-i \eta) t} R(\sigma-i \eta, C)(f)(0) d \sigma .
\end{aligned}
$$

Since $\mid R(\sigma \pm i \eta) C)(f)(0) \mid \rightarrow 0$ as $\eta \rightarrow \infty$ uniformly with respect to $\sigma \in[0, \epsilon]$, it follows that pointwise on $(0, \infty)$

(3.17) $\lim _{n \rightarrow \infty} \int_{-n}^{n} e^{(\epsilon+i \eta) t} R(\epsilon+i \eta, C)(f)(0)=\lim _{n \rightarrow \infty} \int_{-n}^{n} e^{i \eta t} R(i \eta, C)(f)(0) d \eta$.

By (3.14) it follows that the second limit of (3.17) exists a.e. for $t>0$ and it equals $g(t)$. Thus

$$
y(t)=\frac{1}{2 \pi} \int_{-\infty}^{\infty} e^{i \eta t}(\eta i I-A-\hat{B}(i \eta))^{-1}(f(0)+\widehat{B * f}(i \eta)) d \eta \quad \text { a.e. for } t>0
$$

From the Parseval formula, we obtain 


$$
\begin{aligned}
\int_{0}^{\infty}|y(t)|^{2} d t & =\int_{-\infty}^{\infty}\left|(\eta i I-A-\hat{B}(i \eta))^{-1}(f(0)+\widehat{B * f}(i \eta))\right|^{2} d \eta \\
& \leq\left(|f(0)|+\|B\|_{1}\|f\|_{1}\right)^{2} \int_{-\infty}^{\infty}\left|(\eta i I-A-\hat{B}(i \eta))^{-1}\right|^{2} d \eta \text {. Q.E.D. }
\end{aligned}
$$

Corollary 3.9. In addition to the bypotheses of Theorem 2, assume that $f \in$ $L^{2}(-\infty, 0]$. Then the solution $y(t ; f)$ approaches zero as $t \rightarrow \infty$.

Proof. From equation (L), it is apparent that $f \in L^{2}(-\infty, 0], y \in L^{2}[0, \infty)$ and $B \in L^{1}[0, \infty)$ implies that $y^{\prime} \in L^{2}[0, \infty)$. Hence $y(t)$ is uniformly continuous and the result follows.

\section{BIBLIOGRAPHY}

1. R. K. Miller, Asymptotic stability of linear Volterra integro-differential equations, J. Differential Equations (to appear).

2. S. I. Grossman and R. K. Miller, Linear Volterra integro॰differential systems with $L^{1}$ kernels (submitted).

3. E. Hille and R. S. Phillips, Functional analysis and semigroups, rev. ed., Amer. Math. Colloq. Publ., vol. 31, Amer. Math. Soc., Providence, R. I., 1957. MR 19, 664.

4. R. D. Driver, Existence and stability of solutions of a delay-differential system, Arch. Rational Mech. Anal. 10 (1962), 401-426. MR 25 \#5260.

5. J. J. Levin and J. A. Nohel, On a system of integro-differential equations occuring in reactor dynamics, J. Math. Mech. 9 (1960), 347-368. MR $22 \# 8301$.

6. K. Yosida, Functional analysis, Die Grundlehren der math. Wissenschaften, Band 123, Academic Press, New York; Springer-Verlag, Berlin, 1965. MR 31 \#5054.

FACULTY OF MATHEMATICS, UNIVERSITY OF IAȘI, IAȘI, ROMANIA

DEPARTMENT OF MATHEMATICS, McGILl UNIVERSITY, MONTREAL, QUEBEC, CANADA

Current address (S. I. Grossman): Department of Mathematics, University of Montana, Missoula, Montana 59801 